\title{
Ginzburg-Landau theory of superconducting surfaces under electric fields
}

\author{
P. Lipavský1 ${ }^{1,2}$, K. Morawetz ${ }^{3,4}$, J. Koláček ${ }^{2}$, T. J. Yang ${ }^{5}$ \\ ${ }^{1}$ Faculty of Mathematics and Physics, Charles University, Ke Karlovu 3, 12116 Prague 2, Czech Republic \\ ${ }^{2}$ Institute of Physics, Academy of Sciences, Cukrovarnická 10, 16253 Prague 6, Czech Republic \\ ${ }^{3}$ Institute of Physics, Chemnitz University of Technology, 09107 Chemnitz, Germany \\ ${ }^{4}$ Max-Planck-Institute for the Physics of Complex Systems, Noethnitzer Str. 38, 01187 Dresden, Germany \\ ${ }^{5}$ Department of Electrophysics, National Chiao Tung University, Hsinchu 300, Taiwan
}

\begin{abstract}
A boundary condition for the Ginzburg-Landau wave function at surfaces biased by a strong electric field is derived within the de Gennes approach. This condition provides a simple theory of the field effect on the critical temperature of superconducting layers.
\end{abstract}

The critical temperature of a thin superconducting layer is increased or lowered by an electric field applied perpendicular to the layer. ${ }^{1-5}$ Similarly to the conductivity of inverse layers in semiconductors, superconductivity of thin metallic layers can thus be controlled by a gate voltage, which makes these structures attractive for applications.

In this paper we show that the phase transition in a thin metallic layer is conveniently described by the Ginzburg-Landau (GL) theory, where the electric field $E$ enters the GL boundary condition as

$$
\left.\frac{\nabla \psi}{\psi}\right|_{0}=\left.\frac{\nabla \Delta}{\Delta}\right|_{0}=\frac{1}{b}=\frac{1}{b_{0}}+\frac{E}{U_{\mathrm{s}}}
$$

Briefly, the logarithmic derivative of the GL function $\psi$ or the gap function $\Delta$ at the surface is a sum of the zerofield part $1 / b_{0}$ and the field induced correction $E / U_{\mathrm{s}}$.

The zero-field part has been derived by de Gennes ${ }^{6}$ from the BCS theory. A typical value $b_{0} \sim 1 \mathrm{~cm}$ is large on the scale of the GL coherence length, therefore this contribution is usually neglected. This approximation, $1 / b_{0} \approx 0$, corresponds to the original GL condition $\nabla \psi=0$.

Here we employ the de Gennes approach to derive the field induced correction $E / U_{\mathrm{s}}$. The correction becomes important for the above mentioned experiments, where fields of the order of $10^{7} \mathrm{~V} / \mathrm{cm}$ are realized. Small electric fields appearing e.g. in Josephson junctions do not require such corrections.

We start from the condition

$$
\frac{1}{b}=\frac{1}{\xi^{2}(0)} \frac{1}{N_{0} V} \int_{-\infty}^{\infty} d x \frac{\Delta(x)}{\Delta_{0}}\left[1-\frac{N(x)}{N_{0}}\right]
$$

derived by de Gennes (Eq. (7-62) in Ref. 6). Here $N_{0}$ is the density of states of a bulk material, $V$ is the BCS interaction, and $N(x)$ is the local density of states at position $x$. The actual gap function $\Delta(x)$ has a nontrivial profile close to the surface at $x=0$, but it has only slow variation at distances exceeding the BCS coherence length $\xi_{0}=0.18 \hbar v_{\mathrm{F}} / k_{\mathrm{B}} T_{\mathrm{c}}$. For $x \sim \xi_{0}$ it is crudely linear $\Delta(x) \approx \Delta_{0}(1+x / b)$, so that $\Delta_{0}$ is not the true surface value but the extrapolation of the gap function towards the surface. In Eq. (2) we have used the GL coherence length at zero temperature $\xi(0)=0.74 \xi_{0}$ for pure metals.

In measurements of the field effect on the transition temperature, the zero-field term $b_{0}$ is included in the reference zero-bias transition temperature. Accordingly, we can assume a model of the crystal for which $1 / b_{0}=0$. The simplest model of this kind is a semi-infinite jellium, where for zero field the density of states is steplike, $N(x)=N_{0}$ for $x>0$ and $N(x)=0$ elsewhere. Using that the gap function is restricted to the crystal, $\Delta(x)=0$ for $x<0$, one can check that from (2) follows $1 / b_{0}=0$.

Now we include the electric field. According to the Anderson theorem ${ }^{7}$, the electric field does not change the thermodynamical properties directly but only via the density of states. The change of the density of states is also indirect. The penetrating electric field induces a deviation $\delta n$ of the electron density. The density deviation changes the Fermi momentum. Since the density of states depends on the Fermi momentum, its value becomes modified. We express this complicated indirect effect approximatively via a local linear expansion

$$
N(x)=N_{0}+\frac{\partial N_{0}}{\partial n} \delta n(x) .
$$

The de Gennes condition (2) then reads

$$
\frac{E}{U_{\mathrm{s}}}=-\frac{1}{\xi^{2}(0)} \frac{1}{N_{0}^{2} V} \frac{\partial N_{0}}{\partial n} \int_{0}^{\infty} d x \frac{\Delta(x)}{\Delta_{0}} \delta n(x) .
$$

The actual space profile of $\delta n$ in superconductors is unknown. In fact, some of recent measurements suggests that the electric field penetrates deep into superconductors. ${ }^{8}$ Interpretation of these observations is not yet settled, therefore we prefer to assume that the screening in superconductors is similar to the screening in normal metals so that $\delta n$ is non-zero only on the scale of the Thomas-Fermi screening length. The typical Thomas-Fermi length is less then one Angström, while the gap function varies on a scale typical to the BCS kernel $\sim \xi_{0}$. Accordingly, in the integral (4) we can take $\Delta(x) \approx \Delta(0)$ and obtain

$$
\frac{1}{U_{\mathrm{s}}}=\frac{1}{\xi^{2}(0)} \frac{1}{N_{0}^{2} V} \frac{\partial N_{0}}{\partial n} \frac{\Delta(0)}{\Delta_{0}} \frac{\epsilon_{0}}{e} .
$$


In this rearrangement we have used the surface charge determined by the applied field $\epsilon_{0} E=-e \int_{0}^{\infty} d x \delta n(x)$.

The effective potential $U_{\mathrm{s}}$ given by (5) depends on bulk material parameters $\xi_{0}, N_{0} V$ and $\partial N_{0} / \partial n$, and on the ratio of the gap at the surface to the bulk value

$$
\eta=\frac{\Delta(0)}{\Delta_{0}}
$$

According to de Gennes estimates ${ }^{6}$, the surface ratio $\eta$ is of the order of unity. A heuristic derivation of the fieldeffect from the GL equation cannot cover this factor. ${ }^{9}$

It is advantageous to express the effective potential $U_{\mathrm{s}}$ via the usual parameters of the GL theory. First we employ the BCS relation for the critical temperature $k_{\mathrm{B}} T_{\mathrm{c}}=1.14 \hbar \omega_{\mathrm{D}} \exp \left(-1 / N_{0} V\right)$. The critical temperature depends on the density of electrons. Comparing alloys with different impurity doping, it has been deduced that the dominant density dependence enters the critical temperature via the density of states. ${ }^{10}$ We can thus assume $\partial \omega_{\mathrm{D}} / \partial n \approx 0$ and $\partial V / \partial n \approx 0$ with the help of which we express the derivative of the density of states via the logarithmic derivative of the critical temperature. Formula (5) then simplifies to

$$
\frac{1}{U_{\mathrm{s}}}=\eta \kappa^{2} \frac{\partial \ln T_{\mathrm{c}}}{\partial \ln n} \frac{e}{m c^{2}} .
$$

Here we have expressed the electron density via the London penetration depth $\lambda^{2}(0)=m /\left(\mu_{0} n e^{2}\right)$. Its ratio to the GL coherence length defines the GL parameter $\kappa=\lambda(0) / \xi(0)$.

Let us estimate the effective potential $U_{\mathrm{s}}$ for niobium. The charge carriers are electrons, $e=-|e|$, with the mass close to the electron rest mass, $m \approx 1.2 m_{\mathrm{e}}$. The GL parameter is on the edge of the type-I and II materials, $\kappa=0.78$, and the logarithmic derivative is of moderate amplitude, $\partial \ln T_{\mathrm{c}} / \partial \ln n=0.75$ (see Ref. 11). Taking $\eta \approx 1$ one finds, $U_{\mathrm{s}}=-1.310^{6} \mathrm{~V}$. As one can see, a large field $E \sim 10^{6} \mathrm{~V} / \mathrm{cm}$ is necessary to create a fieldinduced correction at least comparable to the commonly neglected zero-field value $1 / b_{0} \sim 1 / \mathrm{cm}$.

The effective potential (7) is the major result of this paper. Now we use it in the boundary condition (1) to evaluate the transition temperature $T^{*}$ of a biased layer of a finite thickness $L$. General steps of our analysis parallel the theory of the Little-Parks effect ${ }^{12}$. It is also in a close analogy to the theory of surface superconductivity in short coherence length materials ${ }^{13}$.

Let us assume that the electric field is applied only to the left surface at $x=0$, while the right surface at $x=L$ is free of the field. We take $1 / b_{0}=0$ for simplicity, so that we use the boundary conditions

$$
\begin{aligned}
& \left.\frac{\nabla \psi}{\psi}\right|_{0}=\frac{E}{U_{\mathrm{s}}}, \\
& \left.\frac{\nabla \psi}{\psi}\right|_{L}=0 .
\end{aligned}
$$

The GL function is given by the dimension-less GL equation $\xi^{2}(T) \nabla^{2} \psi+\psi-|\psi|^{2} \psi=0$, see Ref 12 . The transition point is characterized by an infinitesimally small GL function, $|\psi|^{2} \rightarrow 0$. At the transition temperature $T^{*}$, the non-linear term in the GL equation thus vanishes $\xi^{2}\left(T^{*}\right) \nabla^{2} \psi+\psi=0$. This equation is solved by $\psi(x) \propto \cos \left[(x-L) / \xi\left(T^{*}\right)\right]$, which satisfies the right boundary condition (9) while the left boundary condition (8) demands

$$
\frac{L}{\xi\left(T^{*}\right)} \tan \left(\frac{L}{\xi\left(T^{*}\right)}\right)=\frac{E L}{U_{\mathrm{s}}} .
$$

When the superconductor has a coherence length $\xi$ which satisfies the condition (10), the non-zero GL wave function nucleates and the system undergoes a transition to the superconducting state. Since the coherence length is a function of temperature,

$$
\xi(T)=\frac{\xi(0)}{\sqrt{1-\frac{T}{T_{\mathrm{c}}}}},
$$

one can find from (10) and (11) the transition temperature $T^{*}$. It reads

$$
T^{*}=T_{\mathrm{c}}-T_{\mathrm{c}} \frac{\xi^{2}(0)}{L^{2}} g\left(\frac{E L}{U_{\mathrm{s}}}\right),
$$

where the function $g(\tau)$ is a root of $\sqrt{g} \tan \sqrt{g}=\tau$. The function $g$ is plotted in figure 1 .

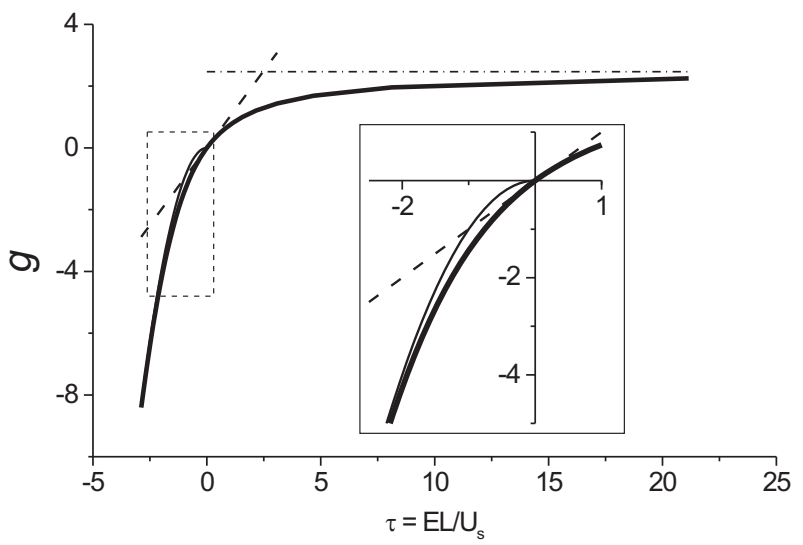

FIG. 1. The dimensionless shift of the transition temperature due to the electric field as given by Eq. (12). The exact solution of $\sqrt{g} \tan \sqrt{g}=\tau$ (thick full line), the linear approximation $g \approx \tau$ for thin layers (tangential dashed line), the constant approximation $g \approx \pi^{2} / 4$ for large suppressive fields (dot-dashed line), and the parabolic approximation $g \approx-\tau^{2}$ for large supportive fields (thin full line). The insert shows a detail of the parabolic approximation.

Although equation (12) is simple by itself, we find it useful to discuss its asymptotic solutions. Let us start with the experimentally most important limit. The effect of the electric field on the transition temperature 
is rather small and it is most conveniently observed on very thin layers. In this case $|E L| \ll\left|U_{s}\right|$ and one can use the linear approximation $g(\tau) \approx \tau$ shown as the dashed line in Fig 1. Within linear approximation the transition temperature (11) simplifies to

$$
T^{*}=T_{\mathrm{c}}-\eta \frac{\partial T_{\mathrm{c}}}{\partial n} \frac{\epsilon_{0} E}{e L} .
$$

Formula (13) shows that surface ratio (6) reduces field effect on the transition temperature $T^{*}$. One can compare (13) with a simple estimate that assumes that the induced charge is homogeneously distributed across the layer, $e \delta n(x) \approx-\epsilon_{0} E / L$. A bulk critical temperature modified by the excess charge is then interpreted as the transition temperature, $T^{*}=T_{\mathrm{c}}+\partial T_{\mathrm{c}} / \partial n \times \delta n$. Apparently, the simple estimate does not include the surface ratio $\eta$.

Based on the simple estimate one is tempted to say that $\partial T_{\mathrm{c}} / \partial n$ can be uniquely determined from experimental data on $T^{*}$. The present theory shows, however, that surface ratio obscures the observed value.

Among high- $T_{\mathrm{c}}$ superconductors there are many materials of large $\kappa$. From equation (7) one can see that these materials have much lower effective potential $U_{\mathrm{s}}$, therefore they reveal a much stronger field effect on the transition temperature. With these materials it is possible to achieve the opposite limit - the regime of thick layers $|E L| \gg\left|U_{\mathrm{s}}\right|$.

A measurement in the regime of thick layers has been already performed by Matijasevic et al. ${ }^{14}$ We will use parameters of their sample to evaluate effects expected from formula (12). The sample $\mathrm{Sm}_{0.7} \mathrm{Ca}_{0.3} \mathrm{Cu}_{3} \mathrm{O}_{y}$ is overdoped with $T_{\mathrm{c}}$ reduced to $50 \mathrm{~K}$. The carriers are holes, $e=|e|$, of the mass $m=3.46 m_{\mathrm{e}}$ and the density $n=5.7510^{21} / \mathrm{cm}^{3}$. Based on the authors claim that $T_{\mathrm{c}}$ in a monolayer would increase by $10 \mathrm{~K}$ at the imposed voltage, one can deduce $\partial \ln T_{\mathrm{c}} / \partial \ln n=-3.12$. With a typical GL parameter $\kappa=100$ one obtains the GL coherence length $\xi(0)=1.3 \mathrm{~nm}$ and an effective potential $U_{\mathrm{s}}=-56.6 \mathrm{~V}$. Note that this is by four orders of magnitude smaller than the niobium value. The applied field is enhanced by a large dielectric function to an effective value $E=7.810^{7} \mathrm{~V} / \mathrm{cm}$. For a sample width of $L=50 \mathrm{~nm}$ and $\eta \approx 1$ we find $\left|E L / U_{\mathrm{s}}\right|=6.9$, which confirms that these measurements are in the thick layer limit.

In the thick layer limit one has to distinguish whether the electric field supports or depresses the transition temperature. Let us first discuss the depression which appears for $\partial T_{\mathrm{c}} / \partial n \times \epsilon_{0} E / e>0$. In this case $E L / U_{\mathrm{s}}>0$ and the function $g$ approaches the constant asymptotic value $g \rightarrow \pi^{2} / 4=2.47$ shown as the dash-dotted line in Fig. 1. Since $g<\pi^{2} / 4$, relation (12) yields the upper estimate of the depression of the transition temperature

$$
T_{\mathrm{c}}-T^{*}<T_{\mathrm{c}} \frac{\pi^{2} \xi^{2}(0)}{4 L^{2}}
$$

One can see that the maximal depression is limited by the layer thickness. Within the adopted approximations the actual value of the electrostatic field does not matter once the asymptotic regime is reached. For parameters of Ref. 14 one finds from formula (14) the lower estimate $T_{\mathrm{c}}-T^{*}<0.08 \mathrm{~K}$. The formula (12) gives a slightly smaller value, as one can guess from Fig. 1. For $\left|E L / U_{\mathrm{s}}\right|=6.9$ the dimensionless shift is $g(6.9)=1.89$, i.e., $T^{*}-T_{\mathrm{c}}=-0.064 \mathrm{~K}$. Matijasevic et $a l^{14}$ reported no suppression of the superconductivity. Our estimate shows that the suppression is below the sensitivity of their method.

A different situation is met if the direction of the electric field is reversed. The electric field then supports the superconductivity since $\partial T_{\mathrm{c}} / \partial n \times \epsilon_{0} E / e<0$. In this case $E L / U_{\mathrm{s}}<0$ and the function $g$ approaches its quadratic asymptotics, $g \rightarrow-\tau^{2}$ shown as the thin full line in Fig. 1. Since $g=L^{2} / \xi^{2}\left(T^{*}\right)$, the coherence length $\xi\left(T^{*}\right)$ is imaginary giving the GL function exponentially decaying from the biased surface. In this limit,

$$
T^{*} \rightarrow T_{\mathrm{c}}+T_{\mathrm{c}} \frac{E^{2} \xi^{2}(0)}{U_{\mathrm{s}}^{2}},
$$

the critical temperature does not depend on the width of sample and increases quadratically with the electric field. For parameters of Ref. 14 we obtain $T^{*}-T_{\mathrm{c}}=1.6 \mathrm{~K}$ in a reasonable agreement with the reported shift by $1 \mathrm{~K}$.

The increased critical temperature (15) is independent of the layer width $L$. This shows that in this limit the superconductivity above $T_{\mathrm{c}}$ is stimulated in the same way as the superconductivity on the surface of an infinite sample predicted by Shapiro ${ }^{15}$. Formula (15) differs from Shapiro's formula (9) only by a factor due to the impurity limited coherence length assumed in Ref. 15.

We note that the present discussion does not account for the charge reservoirs typical to layered $\mathrm{CuO}$ materials. For a microscopic study devoted to these materials see Ref. 16. We also do not assume an eventual effect of the electric field on the chemical composition, e.g., due to oxygen motion as proposed in Refs. 17, 18.

In summary, using the de Gennes approach we have derived the GL boundary condition for a superconducting surface exposed to the electric field. This boundary condition allows one to conveniently evaluate the field effect on surface sensitive phenomena from the GL theory. Its implementation is demonstrated for the field effect on the superconducting phase transition in metallic layers. Our approach recovers known features, in particular, that for thin layers the transition temperature can be linearly enhanced or suppressed depending on the orientation of the applied field. We have found, however, that compared to former theories the linear coefficient is modified by the value of the gap at the surface. In the limit of thick layers we obtain a field induced surface superconductivity with the shift of the critical temperature depending on the square of the electric field. We also obtain the upper limit on the suppression of the critical temperature being 
independent of the field and inversely proportional to the square of the layer width. These features agree with the experimental data.

\section{ACKNOWLEDGMENTS}

Authors are grateful to P. Martinoli who brought this problem to their attention. This work was supported by GAČR 202/04/0585, 202/05/0173, GAAV A1010312 grants, by National Science Council of Republic of China, Taiwan, with grant Nsc 94-2112-M-009-001, and by DAAD project D/03/44436. The European ESF program AQDJJ is also acknowledged.

${ }^{1}$ R. E. Glover and M. D. Sherrill, Phys. Rev. Lett. 5, 248 (1960).

${ }^{2}$ X. X. Xi et al., Phys. Rev. Lett. 68, 1240 (1992).

3 T. Frey, J. Mannhart, J. G. Bednorz, and E. J. Williams, Phys. Rev. B 51, 3257 (1995).

${ }^{4}$ C. H. Ahn, J. M. Triscone, and J. Mannhart, Nature 424, 1015 (2003).

${ }^{5}$ D. Matthey, S. Gariglio, and J. M. Triscone, Appl. Phys. Lett. 83, 3758 (2003).

${ }^{6}$ P. G. de Gennes, Superconductivity of Metals and Alloys (Benjamin, New York, 1966), Chap. VII.3.

${ }^{7}$ P. W. Anderson, J. Phys. Chem. Solids 11, 26 (1959).

${ }^{8}$ R. Tao, X. Xu, and E. Amr, Phys. Rev. B 68, 144505 (2003).

${ }^{9}$ W. D. Lee, J. L. Chen, T. J. Yang, and B.-S. Chiou, Physica C 261, 167 (1996).

${ }^{10}$ C. M. Varma and R. C. Dynes, in Superconductivity in $d$ - and f-band Metals, edited by D. H. Douglass (Plenum Press, New York, 1976).

${ }^{11}$ P. Lipavský, J. Kolacek, K. Morawetz, and E. H. Brandt, Phys. Rev. B 66, 134525 (2002).

12 M. Tinkham, Introduction to Superconductivity (McGraw Hill, New York, 1966), Chap. 4.

13 J. L. Chen and T. J. Yang, Physica C 231, 91 (1994).

${ }^{14}$ V. C. Matijasevic et al., Physica C 235, 2097 (1994).

${ }^{15}$ B. Y. Shapiro, Solid State Commun. 53, 673 (1985).

${ }^{16}$ P. Konsin and B. Sorkin, Phys. Rev. B 58, 5795 (1998).

${ }^{17}$ N. Chandrasekhar, O. T. Valls, and A. M. Goldman, Phys. Rev. Lett. 71, 1079 (1993).

${ }^{18}$ G. Grigelionis, E. E. Tornau, and A. Rosengren, Phys. Rev. B 53, 425 (1996). 